Original Research

\title{
Comparison of the Effects of Static-Stretching and Tubing Exercises on Acute Shoulder Range of Motion in Collegiate Baseball Players
}

\author{
Andrew M Busch, EdD, CSCS $^{1}$, Jackson Browstein ${ }^{1}$, Richard UIm, DC, CSCS $^{2}$ \\ 1 Department of Health and Human Kinetics, Ohio Wesleyan University, ${ }^{2}$ Columbus Chiropractic \& Rehabilitation Center \\ Keywords: static stretching, movement system, glenohumeral joint, dynamic exercise, baseball \\ https://doi.org/10.26603/001c.18862
}

International Journal of Sports Physical Therapy

Vol. 16, Issue 1, 2021

\section{Background}

The overhead throwing motion repetitively stresses the dominant arm in baseball players, frequently altering normal range of motion (ROM) in multiple directions. Baseball players regularly perform a combination of static stretches (SS) and dynamic tubing (DT) resistance exercises in pre-throwing warm-up routines intended to improve shoulder ROM and reduce injuries.

\section{Purpose}

The purpose of this study was to compare acute changes in dominant shoulder ROM improvements between SS and DT warm-up exercise protocols. The DT exercises were hypothesized to elicit greater improvements in shoulder ROM.

\section{Study Design}

Two-way crossover study.

\section{Methods}

Twenty-five healthy collegiate baseball players (mean age $=19.8 \pm 1.0$ years) presenting with glenohumeral internal rotation deficit (GIRD) $>20^{\circ}$ and total rotational range of motion (TRROM) losses $>5^{\circ}$ completed the SS and DT interventions on different days. Dominant arm internal rotation (IR), external rotation (ER) and TRROM were measured before, immediately after, 30-minutes after, and 60-minutes after each treatment session. A two-way repeated measures analysis of variance (ANOVA) compared the effect of SS and DT over time on IR, ER and TRROM.

\section{Results}

IR improved on average $10.68 \pm 0.82^{\circ}(\mathrm{p}<.001)$ post intervention, $11.18 \pm 0.79^{\circ}(\mathrm{p}<.001)$ 30 -min post intervention, and $9.03 \pm 0.95^{\circ}(\mathrm{p}<.001) 60$-min post intervention. ER improved on average $8.60 \pm 0.67^{\circ}(p<.001)$ post intervention, $8.25 \pm 0.85^{\circ}(p<.001)$ 30 -min post intervention, and $6.65 \pm 0.91^{\circ}(\mathrm{p}<.001) 60$-min post intervention. TRROM improved on average $19.28 \pm 1.09^{\circ}(\mathrm{p}<.001)$ post intervention, $19.43 \pm 1.36^{\circ}(\mathrm{p}<.001)$ 30 -min post intervention, and $15.68 \pm 1.55^{\circ}(\mathrm{p}<.001) 60$-min post intervention. There were no significant differences between the main effects of treatment and time for IR, ER, and TRROM. For IR, SS improved by an average of $1.73 \pm 0.55^{\circ}(\mathrm{p}=.005)$ more than DT. For ER and TRROM, there were no differences between SS and DT.

\footnotetext{
a Corresponding author: Andrew M Busch 107C Edwards Gymnasium 61 S. Sandusky St. Delaware OH 43015 Phone: (740) 368-3864 ambusch@owu.edu
} 


\section{Conclusion}

Both SS and DT exercises improve glenohumeral IR, ER and TRROM up to one-hour post intervention, with no significant differences noted between interventions for treatment or time. Baseball players can benefit equally from performing SS or DT exercises to acutely improve shoulder ROM.

\section{Level of Evidence}

Level 3

\section{INTRODUCTION}

The baseball throwing motion repetitively creates large translational forces and rotational moments on the shoulder and elbow as the arm dynamically moves through susceptible end-range positions. ${ }^{1}$ It has been well established in studies investigating shoulder range of motion (ROM) in baseball players that differences exist between arms, such that external rotation (ER) tends to increase while internal rotation (IR) typically decreases in the dominant arm compared to the non-dominant arm. ${ }^{2-4}$ One common adaptation is known as glenohumeral internal rotation deficit (GIRD) $)^{3,5}$ which is the excessive loss of IR in the glenohumeral joint compared to the opposite arm. ${ }^{5-7}$ Bilateral differences of $10-15^{\circ}$ are considered normal for IR or $\mathrm{ER}^{8}$, whereas deficits $>20^{\circ}$ are a cause for concern and increase injury risk in baseball players. ${ }^{5,9}$ In addition to GIRD, total rotational range of motion (TRROM), the sum of glenohumeral IR and ER, has consistently been demonstrated to fall within $5^{\circ}$ bilaterally ${ }^{8}$ and thought to provide even greater relevance when assessing shoulder ROM in baseball players within clinical settings. ${ }^{9}$ It is unclear however which measurement is more important when attempting to reduce injuries.

Alterations of shoulder ROM in response to throwing are multifactorial, as bony and soft tissue adaptations contribute to commonly observed changes. ${ }^{8}$ Bone tissue can adapt to repetitive loading, and two common adaptations include increased humeral retrotorsion (HRT), which is the angle of rotation in the epiphyseal axis relative to the greater and lesser humeral tubercles, $, 8,10$ and increased glenohumeral retroversion (GRV) ${ }^{8}$ which is the angle subtended by the glenoid line relative to a perpendicular scapular line on the posterior aspect of the scapula. ${ }^{11}$ Collegiate baseball players exhibit greater HRT in their dominant arms when compared to age-matched controls with no history of overhead sport participation. ${ }^{12}$ Professional pitchers who present with GIRD have also displayed greater side-to-side differences in HRT when compared to pitchers without GIRD,$^{3}$ and those who demonstrate $>20^{\circ}$ GIRD are twice as likely to become injured compared to pitchers without GIRD. ${ }^{13}$ The relationship of increased HRT and GRV are not fully understood, but seem to be coupled during maturation.

Soft tissue adaptations can also occur in the capsule or muscular structures of the shoulder. ${ }^{14}$ The repetitive stress throwing places on the anterior shoulder capsule is theorized to lead to tightening of the posterior capsule. ${ }^{15} \mathrm{Sev}$ eral studies have revealed patients who present with injuries such as labral tears and impingement-syndrome also demonstrate GIRD and tight posterioinferior shoulder cap- sules. ${ }^{5,13,16}$ In addition to capsular thickening, a shortening response and stiffening of the rotator cuff muscles can occur due to the exposure history of those muscles with throwing. ${ }^{8,14}$

A recent systematic review of randomized controlled trials of studies to improve GIRD and posterior shoulder tightness, found that most stretching interventions only performed a single intervention. Six articles only utilized passive stretching, four utilized active stretching, two compared passive to active stretching, and four articles used control groups with no interventions. ${ }^{17}$ While passive and active stretching have been shown to acutely improve shoulder ROM, baseball players tend to perform more dynamic exercises immediately prior to competition, and performing dynamic exercises after a pitching session have been shown beneficial in restoring normal shoulder ROM in professional pitchers. ${ }^{4}$ Much of the research aimed to improve shoulder ROM typically measure only pre- and postintervention, yet it is unclear how long improvements in ROM last after completing the different interventions used. Understanding the duration ROM improvements last would help coaches and athletes optimally time warm-up routines prior to competition. The purpose of this research was to compare acute changes in IR and TRROM improvement between commonly performed static stretches (SS) and dynamic tubing (DT) exercises in the dominant shoulders of collegiate baseball players. A crossover design was utilized to compare three post-intervention measurements over the course of one hour. It was hypothesized that DT exercises would elicit a greater overall improvement in shoulder ROM for both IR and TRROM.

\section{METHODS}

\section{PARTICIPANTS}

A sample of twenty-five healthy male NCAA Division III collegiate baseball players (age $=19.8 \pm 1.0$ years) were recruited from a local university (descriptive statistics are provided in Table 1). All participants were listed on the active roster, and free of injury at the time of screening. Participants were excluded from the study if they had reported a previous elbow or shoulder surgery within the past 12 months. Distribution of the participants by position and dominant arm were: pitchers $(n=11)$, catchers $(n=3)$, infielders $(n=5)$, outfielders $(n=6)$, right-handed $(n=22,88 \%)$, lefthanded ( $n=3,12 \%)$. The academic year of the participants were: freshmen $(n=9)$, sophomores $(n=9)$, juniors $(n=6)$, and seniors $(n=1)$. All participants provided written informed consent prior to testing, and the study was approved a university institutional review board: IRB Protocol \# 1902.004.

All participants received an email with a detailed de- 
Table 1: Descriptive statistics of participants (Mean \pm Standard Deviation)

\begin{tabular}{lccc}
\hline Participants & Age $(\mathrm{y})$ & Height $(\mathrm{cm})$ & Weight $(\mathrm{kg})$ \\
\hline All $(\mathrm{n}=25)$ & $19.8 \pm 1.0$ & $181.8 \pm 4.4$ & $84.4 \pm 7.1$ \\
Pitchers $(\mathrm{n}=14)$ & $19.4 \pm 0.9$ & $181.9 \pm 4.8$ & $86.8 \pm 7.5$ \\
Position Players $(\mathrm{n}=11)$ & $20.3 \pm 1.1$ & $181.5 \pm 4.0$ & $81.2 \pm 5.3$ \\
\hline
\end{tabular}

scription of the study, and completed an athletic and injury history questionnaire prior to testing. Participants met at a university exercise lab on two days, separated by one week during the spring 2019 preseason. At the time of the study, the baseball team had been practicing for 5 weeks, and the competitive season was set to begin in 2 weeks. All pitchers were tested at least two days after throwing off a mound, to prevent any delayed soreness or stiffness from affecting results. Upon arrival the first day, participants were randomly assigned to first perform either the static stretching (SS) intervention, or the dynamic tubing (DT) exercise intervention, and completed the opposite protocol the second day of testing. All participants refrained from throwing prior to data collection on testing days, and were instructed to simply wait patiently for each measurement time during the post-intervention hour.

\section{SHOULDER RANGE OF MOTION ASSESSMENT}

Glenohumeral IR and ER was passively measured for each participant lying supine on an examination table (Figure 1). TRROM was calculated from the sum of IR and ER values. A single examiner collected all measures using a digital inclinometer (model ACU001: Lafayette Instrument Company, Lafayette, IN), while a second examiner stabilized the scapula at the coracoid process with the shoulder abducted at $90^{\circ}$. The examiners were blinded to which intervention the participants were assigned. Intrarater reliability for ROM measures were established on 10 individuals a priori. Strong intraclass correlation coefficients (ICC's) were demonstrated for both $\operatorname{IR}\left(\operatorname{ICC}_{(3,1)}=0.98, \mathrm{SEM}=1.36,95 \% \mathrm{CI}\right.$ $=0.956-0.993)$ and $\operatorname{ER}\left(\operatorname{ICC}_{(3,1)}=0.96, \mathrm{SEM}=1.10,95 \% \mathrm{CI}=\right.$ 0.904-0.984).

Baseline glenohumeral IR and ER was initially measured on both testing days in both arms prior to the interventions, to determine ROM deficits. Three post-intervention measurements were collected: immediately after, 30-minutes post, and 60-minutes post-intervention (Figure 2). All ROM measures post-intervention were only collected in the dominant arms.

\section{STATIC STRETCHING}

The static stretching intervention included: Shoulder extension, doorway stretch, shoulder flexion, cross-body stretch, overhead triceps, and IR @ 90 stretch. These exercises were chosen due to their practicality for on-the-field recommendations because they do not require athletes to lay on the ground or a table. The cross-body stretch has commonly been in research when targeting posterior shoulder tightness and GIRD, and has demonstrated effectiveness in multiple studies. ${ }^{17}$ The IR @ 90 stretch was used
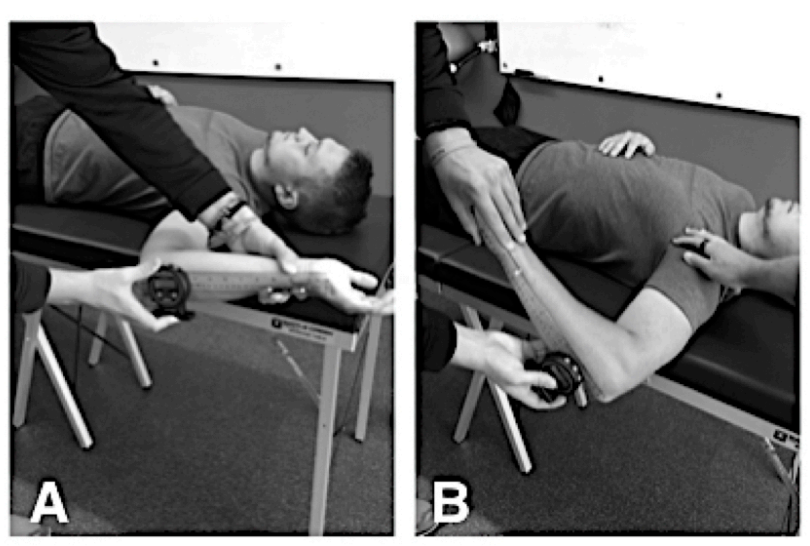

Figure 1: Glenohumeral range of motion assessment. A, External rotation. B, Internal rotation.

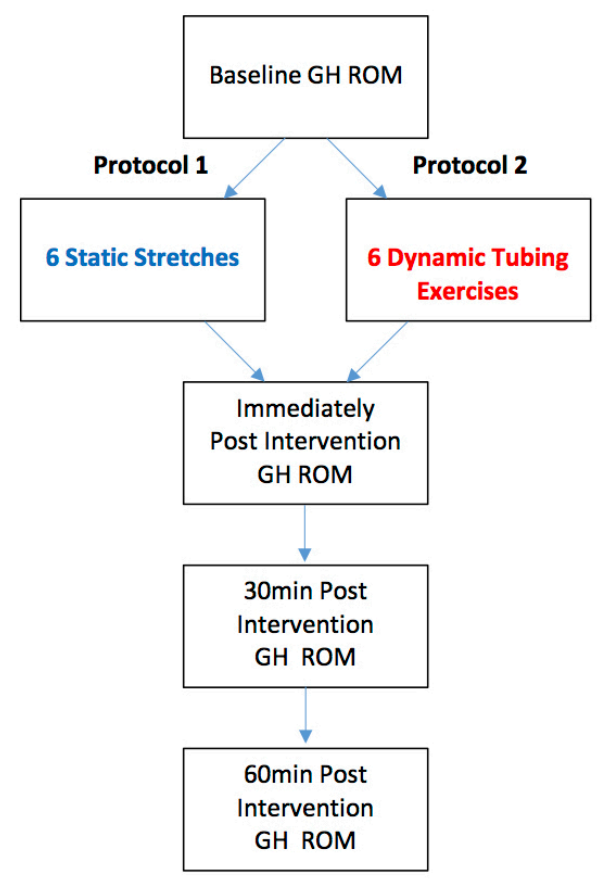

Figure 2: Summary of testing procedures for glenohumeral (GH) ROM

to target IR, and was described by Escamilla et al. ${ }^{4}$ The remaining four stretches: doorway, overhead triceps, flexion $\&$ extension were utilized due to their common use among 
Table 2: Dominant arm glenohumeral range of motion (ROM) for static stretching and dynamic tubing interventions. All are reported in degrees.

\begin{tabular}{|c|c|c|c|c|c|c|c|}
\hline Measure & Intervention & $\begin{array}{c}\text { Baseline } \\
\text { mean } \\
\text { (SD) }\end{array}$ & $\begin{array}{c}\text { Post } \\
\text { Intervention } \\
\text { mean (SD) }\end{array}$ & $\begin{array}{c}\text { 30-min Post } \\
\text { Intervention } \\
\text { mean (SD) }\end{array}$ & $\begin{array}{c}\text { 60-min Post } \\
\text { Intervention } \\
\text { mean (SD) }\end{array}$ & $\begin{array}{l}\text { Effect } \\
\text { Size** }\end{array}$ & $\begin{array}{c}p- \\
\text { Value }\end{array}$ \\
\hline \multirow[t]{2}{*}{$\begin{array}{l}\text { Internal } \\
\text { Rotation }\end{array}$} & $\begin{array}{c}\text { Static } \\
\text { Stretching }\end{array}$ & $\begin{array}{c}49.0 \\
(6.27)\end{array}$ & $60.28(6.15)$ & $60.78(4.19)$ & $59.39(4.77)$ & 0.742 & $<.001^{*}$ \\
\hline & $\begin{array}{l}\text { Dynamic } \\
\text { Tubing }\end{array}$ & $\begin{array}{l}48.55 \\
(6.98)\end{array}$ & $58.63(6.22)$ & $59.13(5.96)$ & $56.23(4.83)$ & 0.758 & $<.001^{*}$ \\
\hline \multirow[t]{2}{*}{$\begin{array}{l}\text { External } \\
\text { Rotation }\end{array}$} & $\begin{array}{c}\text { Static } \\
\text { Stretching }\end{array}$ & $\begin{array}{l}89.91 \\
(6.84)\end{array}$ & 97.92 (5.37) & $97.0(4.99)$ & 95.49 (4.14) & 0.568 & $<.001^{*}$ \\
\hline & $\begin{array}{l}\text { Dynamic } \\
\text { Tubing }\end{array}$ & $\begin{array}{l}90.31 \\
(6.64)\end{array}$ & 99.49 (5.68) & 99.71 (4.87) & 98.03 (4.91) & 0.774 & $<.001^{*}$ \\
\hline \multirow[t]{2}{*}{ TRROM } & $\begin{array}{c}\text { Static } \\
\text { Stretching }\end{array}$ & $\begin{array}{l}138.91 \\
(10.88)\end{array}$ & $\begin{array}{l}158.20 \\
(9.47)\end{array}$ & $157.78(7.07)$ & $154.88(7.58)$ & 0.786 & $<.001^{*}$ \\
\hline & $\begin{array}{l}\text { Dynamic } \\
\text { Tubing }\end{array}$ & $\begin{array}{l}138.85 \\
(11.76)\end{array}$ & $\begin{array}{c}158.12 \\
(9.29)\end{array}$ & $158.84(7.8)$ & $154.25(6.48)$ & 0.841 & $<.001^{*}$ \\
\hline
\end{tabular}

TRRM= total rotational range of motion. Note: Values are degrees of passive ROM

*Statistically significantly difference observed $(p<.05)$

**:Effect size $=$ Partial eta squared $\left(n^{2}\right)$

baseball players. Participants were instructed to hold each position where they felt a strong stretch for a total of $30 \mathrm{sec}-$ onds (Figure 3).

\section{DYNAMIC TUBING EXERCISES}

Baseball players commonly perform many different rubbertubing resistance exercises as part of their warm up routines in attempt to prevent throwing-related injuries. These exercises are usually performed in the dugout or bullpen prior to throwing. The six exercises chosen included: ER at $90^{\circ}$ of abduction, shoulder flexion, shoulder extension, throwing acceleration, throwing deceleration, and low-scapular rows. These specific exercises were chosen due to research previously demonstrating that moderate electromyography (EMG) activation occurs in a majority of the muscles responsible for throwing (rotator cuff, scapular stabilizers, and primary humeral head movers) during those exercises. ${ }^{18}$ Participants completed 10 repetitions of each exercise at a moderate, self-selected tempo (Figure 4).

\section{STATISTICAL ANALYSIS}

Descriptive statistics were calculated for all variables. Data are mean \pm standard deviation, unless otherwise stated. A two-way repeated measures analysis of variance (ANOVA) was run to determine the effect of SS and DT over time on IR and TRROM. Main effects were run for treatment and time, and post hoc tests when necessary. Data analysis showed there was normality, as assessed by the Shapiro-Wilk test of normality with no outliers, as assessed by no studentized residuals greater than \pm 3 standard deviations. Power analysis, using $G^{*}$ Power 3.1 , indicated an $90 \%$ chance of detecting a medium effect size of 0.5 in 10 subjects with statistical significance determined $a$ priori at $p<0.05$ (one tailed). Data analyses were conducted with the Statistical Package for the Social Sciences (SPSS version 26.0 for Mac; IMB Corp, Armonk, NY).

\section{RESULTS}

There was no history of shoulder or elbow surgeries in any of the subjects reported in the previous 12 months. Descriptive statistics are presented for all shoulder ROM data in Table 2. Data are mean \pm standard deviation, unless otherwise stated. There were no statistically significant interactions between treatment and time for IR: $(F(3,72)=1.93$, $\mathrm{p}=.132)$, ER: $(F(1.59,38.08)=2.37, \mathrm{p}=.118)$, or TRROM: $(F(2.18,52.43)=0.48, \mathrm{p}=0.64)$. Therefore, main effects are reported. 
For IR, the main effect of time showed a statistically significant difference between time points, $(F(3,72)=120$, p $<.001)$. Post hoc analyses revealed improvements in ROM on average $10.68 \pm 0.82^{\circ}(95 \% \mathrm{CI}, 8.32$ to $13.04, \mathrm{p}<.001)$ post intervention, $11.18 \pm 0.79^{\circ}(95 \% \mathrm{CI}, 8.91$ to 13.46 , $\mathrm{p}<$ $.001) 30$ - $\min$ post intervention, and $9.03 \pm 0.95^{\circ}(95 \% \mathrm{CI}$, 6.31 to $11.76, \mathrm{p}<.001$ ) 60 -min post intervention (Figure 5). The main effect of treatment also showed a statistically significant difference between SS and DT, $(F(1,24)=9.77, \mathrm{p}=$ .005). Post hoc analyses revealed SS improved by an average $1.73 \pm 0.55^{\circ}$ (95\% CI, 0.58 to 2.87 ), $\mathrm{p}=.005$ more than DT.

For ER, the main effect of time showed a statistically significant difference between time points, $(F(1.47,35.33)=$ $77.72, \mathrm{p}<.001)$. Post hoc analyses revealed improvements in ROM on average $8.60 \pm 0.67^{\circ}$ (95\% CI, 6.66 to $10.54, \mathrm{p}<$ .001 ) post intervention, $8.25 \pm 0.85^{\circ}$ (95\% CI, 5.80 to 10.70 , $\mathrm{p}<.001$ ) 30-min post intervention, and $6.65 \pm 0.91^{\circ}(95 \%$ CI, 4.04 to $9.72, \mathrm{p}<.001$ ) 60 -min post intervention (Figure 6). The main effect of treatment did not show a statistically significant difference between SS and DT, $(F(1,24)=3.04$, p $=.09)$.

For TRROM, the main effect of time showed a statistically significant difference between time points, $(F(1.39$, $33.39)=152.84, \mathrm{p}<.001)$. Post hoc analyses revealed improvements in ROM on average $19.28 \pm 1.09^{\circ}$ (95\% CI, 16.14 to $22.43, \mathrm{p}<.001$ ) post intervention, $19.43 \pm 1.36^{\circ}$ (95\% CI, 15.51 to $23.34, \mathrm{p}<.001$ ) 30 -min post intervention, and $15.68 \pm 1.55^{\circ}$ (95\% CI, 11.22 to $\left.20.15, \mathrm{p}<.001\right) 60$-min post intervention (Figure 6). The main effect of treatment did not show a statistically significant difference between SS and DT, $(F(1,24)=0.003, \mathrm{p}=0.96)$.

\section{DISCUSSION}

The results of this study support the use of both SS and DT exercises as part of a pre-throwing warm-up routine in collegiate baseball players. These findings demonstrate acute changes can be experienced in shoulder ROM through different methods, which can last up to one hour post intervention.

Even though significant IR, ER and TRROM improvements were noted in both interventions, the amount of IR improvement was not enough to categorically de-classify any participant from having GIRD (commonly suggested as $>20^{\circ}$ deficit). ${ }^{2,5,9}$ There was a statistically significant difference between interventions for IR, as the SS intervention showed slightly greater mean improvement compared to DT across all post-measurements, but the difference of only $1.73 \pm 0.55^{\circ}$ is probably not clinically relevant due to the amount of GIRD that was present at baseline. The mean baseline measurements for IR were $49.0 \pm 6.27^{\circ}$ on the SS day, and $48.55 \pm 6.98^{\circ}$ on the DT day. Despite substantial improvements during both interventions, all participants still retained $>20^{\circ}$ IR deficits during all post-intervention measurements. However, the large increases observed in TRROM was enough to de-classify many as 'at-risk', due to the normalization of the dominant arm TRROM compared to the non-dominant arm (suggested as $<5^{\circ}$ deficit). The de-classification of at-risk participants using TRROM was most likely due to the improvement in ER with both interventions. The common measurements used to classify

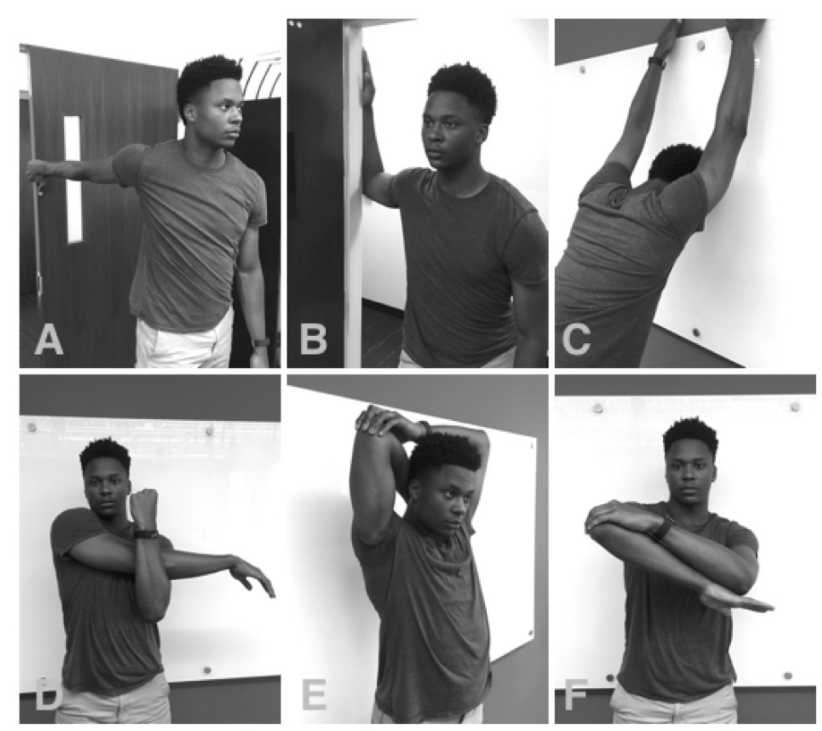

Figure 3: Static stretching intervention. Each stretch was held for a total of $\mathbf{3 0}$ seconds. A, Extension. B, Doorway stretch. C, Flexion. D, Crossbody stretch. E, Overhead triceps. F, Internal Rotation at $90^{\circ}$.

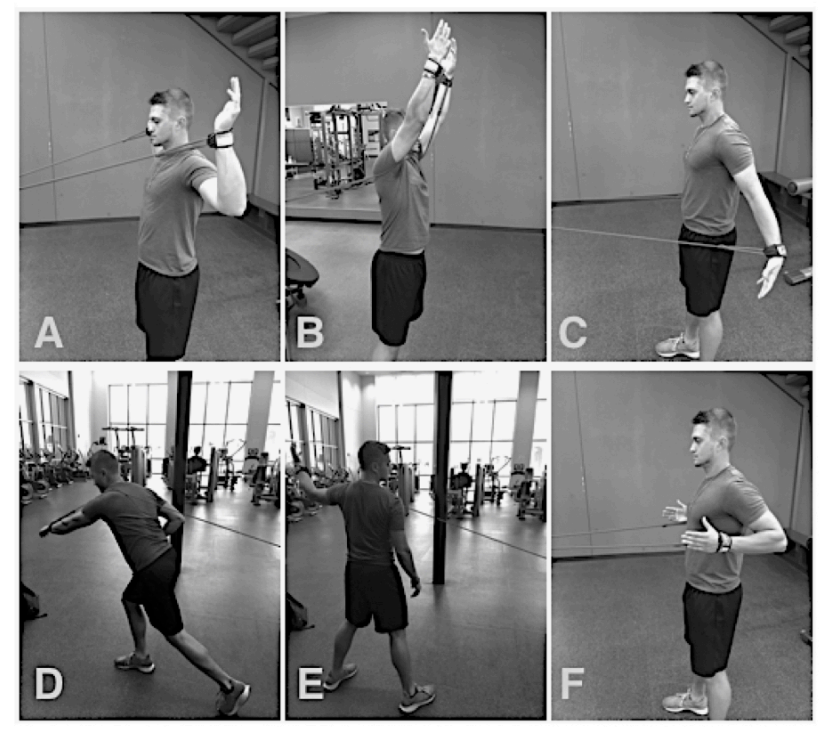

Figure 4: Dynamic tubing intervention. Each exercise was performed for 10 repetitions. $A$, External rotation at $90^{\circ} . \mathrm{B}$, Flexion. C, Extension. D, Acceleration. E, Deceleration. F, Low rows.

injury risk have clinically shown different findings, which can change how a clinician interprets injury risk based on shoulder ROM differences. ${ }^{9}$ These findings illustrate the difficulty often noted when attempting to classify injury risk in shoulders of overhead athletes. One way to strengthen the interpretation of shoulder rotational values is to account for HRT in addition to IR, ER and TRROM, which could better explain clinical findings, yet the use of ultrasound or other imaging may not always be an option 
for clinicians or team trainers.

There are several mechanisms possibly responsible for the ROM adaptations observed in the dominant arm of baseball players. It has often been suggested that decreased IR results from posterior capsular tightness, however, there is evidence demonstrating no significant differences exist in glenohumeral posterior translation between dominant and non-dominant arms in professional baseball players. 19,20 A more likely explanation for why IR deficits remained, despite significant improvements, have to do with osseous adaptations leading to increased HRT and GRV. It is common for athletes across a range of overhead sports to have on average $12^{\circ}$ more HRT in their dominant arm compared to their non-dominant arms. ${ }^{9}$ At birth, the humeral head of both arms are actually in marked HRT, and the normal maturation process involves a period of de-rotation of the humeral head during the pediatric and adolescent years, most rapidly occurring from $0-8$ years. ${ }^{21}$ The asymmetrical retrotorsion commonly seen in adult baseball players is believed to exist from greater throwing stress placed on the shoulder during this skeletally immature time period, while growth plates are likely open. ${ }^{2,21}$ In collegiate pitchers with a history of elbow injuries, greater limb difference in HRT (mean of $7.2^{\circ}$ ) has been noted compared to pitchers with no history of injuries. ${ }^{12}$ While improvements in bony adaptations such as HRT or GRV can only be addressed through surgery, soft-tissue adaptations from throwing did seem to acutely improve in participants of this study, however it is unknown whether those changes were due more to capsular or muscular properties.

Completing SS before athletic performances involving muscular strength and power has thought to exhibit detrimental effects due to altered length-tension relationships, decreased motor unit activation, and reduced muscle spindle activity. ${ }^{22-24}$ In lower body muscles, where most research literature focuses, static stretching can negatively affect performance outcomes in comparison to dynamic exercises. ${ }^{25-27}$ However, adverse effects of SS may be negated when coupled with dynamic activity. ${ }^{22,27,28}$ In a few studies measuring upper body performance, Torres et $\mathrm{al}^{29}$ found no short-term effect of stretching on upper body muscular performance, regardless of stretch type, which may be due to allowing a minimum of five minutes or longer after stretching. Knudson et $\mathrm{al}^{30}$ found no significant effect of SS on the overhand tennis serve when measuring velocity and accuracy in various ages and ability levels. Similarly, a study investigating specifically DIII baseball players, the same population used in the current study, found no differences in average velocity or maximal pitch velocity after performing six different SS, which suggests that acute SS does not deleteriously affect pitching performance. ${ }^{31}$

The improvement in IR in the SS group is most likely due to biomechanical mechanisms such as changes in viscoelastic properties of the muscle-tendon-unit (MTU). ${ }^{32-34}$ The similar improvement observed in the DT group may come from multiple neurological mechanisms. There is likely a level of post-activation potentiation (PAP), wherein activation of the muscles involved in the tested movement improves its contractile performance and function. ${ }^{35}$ Alternatively, it could be due to improved motor control in the shoulder, secondary to increased proprioception, as im-

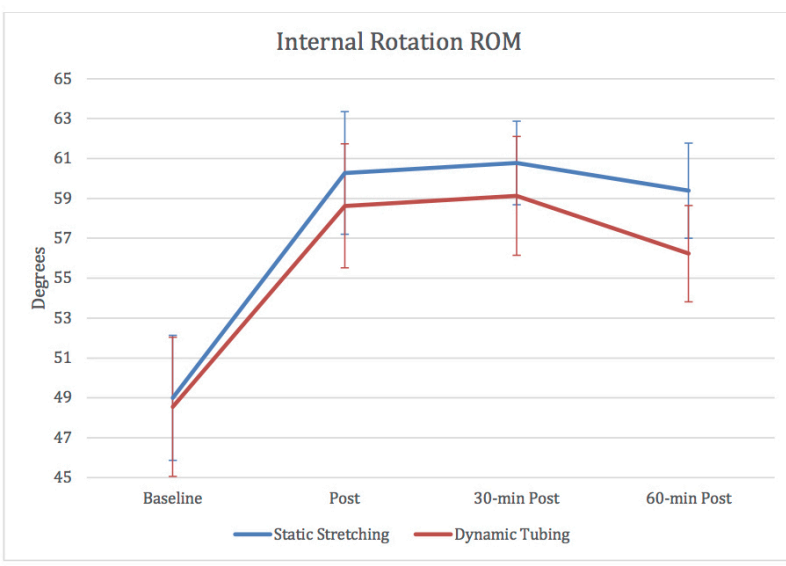

Figure 5: Internal rotation range of motion values for all time measurements.

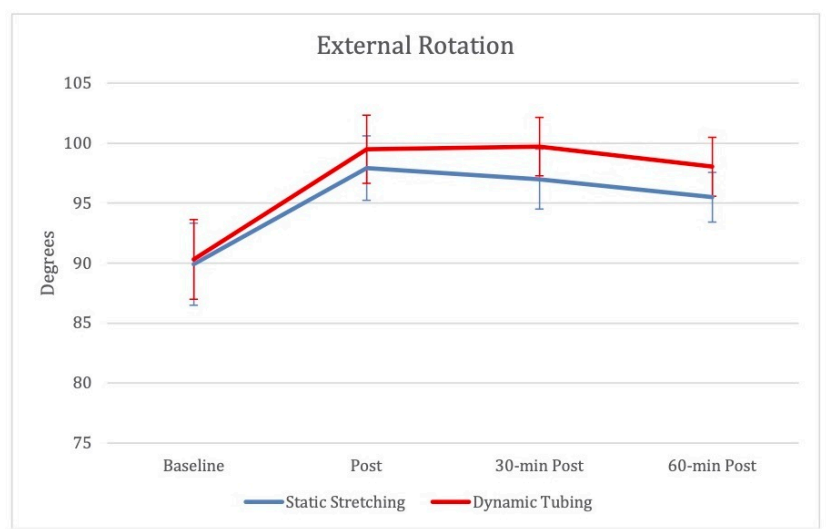

Figure 6: External rotation range of motion values for all time measurements.

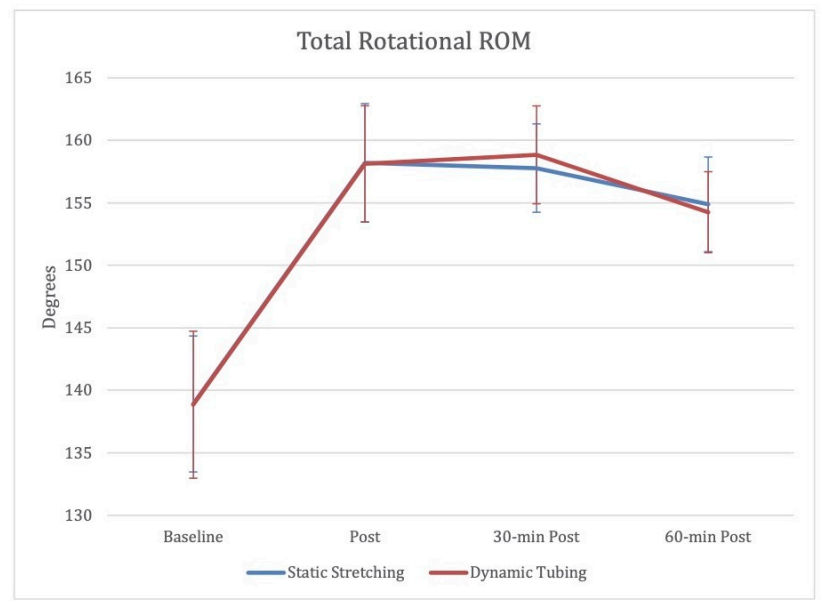

Figure 7: Total rotational range of motion values for all time measurements.

provements in hip ROM have been demonstrated in the absence of stretching, simply through the use of stabilization 
exercises. ${ }^{36}$ Another explanation for improved ROM could be due to proximal stability of the scapula. If instability exists within the shoulder, possibly from inadequate strength in muscles responsible for deceleration during throwing, past injuries, overuse, etc., the shoulder may be protectively guarded by neurologically tightening the posterioinferior capsule. Such guarding could become the symptomatic tight capsule and measurable GIRD. Shoulder rehabilitation protocols have long focused on these aspects of improving shoulder strength and endurance through exercises for the scapular stabilizers and rotator cuff muscles. ${ }^{37}$ By focusing on neuromuscular control during the DT exercises, appropriate proximal stabilizing strategies may have been reinforced. These findings seem to demonstrate some unappreciated neurological components involved in dynamic ROM.

This study is not without limitations. Strength-training routines, and the amount of throwing in the days prior to testing was not recorded. Although pitchers were required to have a minimum of two days' rest between their last bullpen session and each testing day, throwing loads could have varied in each participant between testing days. It is unknown at what point in time, beyond one-hour, ROM improvements possibly return to baseline in this sample. Baseball players often perform both types of the interventions used in this study as part of normal warm-up routines; yet further research is warranted to determine if the order in which they perform such exercises matter. Future research should seek to better understand these effects on upper body performance, as most objective performance tasks measured (pitch speed/ serve speed) are full-body, multijoint movements where the integration of lower body and core muscles may compensate for small upper body changes that possibly occur within the muscle. The effects of longterm stretching outcomes on shoulder ROM is unknown, along with different dosing of the SS and DT exercises, or even a combination of both protocols could possibly result in greater ROM improvements.

\section{CONCLUSIONS}

After completing both SS and DT exercises, passive glenohumeral IR, ER and TRROM can improve for up to onehour in collegiate baseball players exhibiting shoulder ROM deficits. Both interventions demonstrated statistically significant mean improvements for IR, ER and TRROM in all three post-intervention measurements. The SS intervention demonstrated a greater mean improvement $(1.73 \pm$ $0.55^{\circ}$ ) for IR than DT, although probably not clinically significant, as neither intervention improved IR enough to reduce GIRD. There were no differences in the mean improvements in ER or TRROM between the SS and DT groups. This data extends knowledge for therapists, trainers, coaches and athletes who can incorporate either type of warm-up drills to improve shoulder ROM in a time-efficient manner.

\section{ETHICAL STATEMENT}

This study was approved by the Ohio Wesleyan University institutional review board: IRB Protocol \# 1902.004, and all participants signed an informed consent prior to testing.

\section{CONFLICT OF INTEREST AND SOURCE OF FUNDING}

There are no conflicts of interest to report and no funding was received for this study.

Submitted: April 04, 2020 CDT, Accepted: October 10, 2020

CDT 


\section{REFERENCES}

1. Dillman CJ, Fleisig GS, Andrews JR. Biomechanics of pitching with emphasis upon shoulder kinematics. J Orthop Sports Phys Ther. 1993;18(2):402-408.

2. Shanley E, Rauh MJ, Michener LA, Ellenbecker TS, Garrison JC, Thigpen CA. Shoulder Range of Motion Measures as Risk Factors for Shoulder and Elbow Injuries in High School Softball and Baseball Players. Am J Sports Med. 2011;39(9):1997-2006.

3. Noonan TJ, Shanley E, Bailey LB, et al. Professional Pitchers With Glenohumeral Internal Rotation Deficit (GIRD) Display Greater Humeral Retrotorsion Than Pitchers Without GIRD. Am J Sports Med. 2015;43(6):1448-1454. doi:10.1177/036354651557502 $\underline{0}$

4. Escamilla RF, Yamashiro K, Mikla T, Collins J, Lieppman K, Andrews JR. Effects of a Short-Duration Stretching Drill After Pitching on Elbow and Shoulder Range of Motion in Professional Baseball Pitchers. Am J Sports Med. 2017;45(3):692-700. doi:10.1177/036 3546516671943

5. Burkhart SS, Morgan CD, Kibler WB. The disabled throwing shoulder: spectrum of pathology Part I: pathoanatomy and biomechanics. Arthrosc J Arthrosc Relat Surg. 2003;19(4):404-420.

6. Crockett H c., Gross L b., Wilk K e., et al. Osseous adaptation and range of motion at the glenohumeral joint in professional baseball pitchers. / Adaptation osseuse et amplitude des mouvements de l' articulation gleno-humerale chez des lanceurs professionnels de base-ball. American Journal of Sports Medicine. 2002;30(1):20-26.

7. Shanley E, Thigpen CA, Clark JC, et al. Changes in passive range of motion and development of glenohumeral internal rotation deficit (GIRD) in the professional pitching shoulder between spring training in two consecutive years. J Shoulder Elbow Surg. 2012;21(11):1605-1612. doi:10.1016/j.jse.2011.1 $\underline{1.035}$

8. Hellem A, Shirley M, Schilaty N, Dahm D. Review of Shoulder Range of Motion in the Throwing Athlete: Distinguishing Normal Adaptations from Pathologic Deficits. Curr Rev Musculoskelet Med. 2019;12(3):346-355. doi:10.1007/s12178-019-09563-5

9. Whiteley R, Oceguera M. GIRD, TRROM, and humeral torsion-based classification of shoulder risk in throwing athletes are not in agreement and should not be used interchangeably. J Sci Med Sport. 2016;19(10):816-819. doi:10.1016/j.jsams.2015.12.51
10. Sabick MB, Kim Y-K, Torry MR, Keirns MA, Hawkins RJ. Biomechanics of the shoulder in youth baseball pitchers: implications for the development of proximal humeral epiphysiolysis and humeral retrotorsion. Am J Sports Med. 2005;33(11):1716-1722.

11. van de Bunt F, Pearl ML, Lee EK, Peng L, Didomenico P. Glenoid version by CT scan: an analysis of clinical measurement error and introduction of a protocol to reduce variability. Skeletal Radiol. 2015;44(11):1627-1635. doi:10.1007/s 00256-015-2207-4

12. Myers JB, Oyama S, Rucinski TJ, Creighton RA. Humeral Retrotorsion in Collegiate Baseball Pitchers With Throwing-Related Upper Extremity Injury History. Sports Health. 2011;3(4):383-389.

13. Wilk KE, Macrina LC, Fleisig GS, et al. Correlation of Glenohumeral Internal Rotation Deficit and Total Rotational Motion to Shoulder Injuries in Professional Baseball Pitchers. Am J Sports Med. 2011;39(2):329-335. doi:10.1177/0363546510384223

14. Tokish JM. Acquired and adaptive changes in the throwing athlete: implications on the disabled throwing shoulder. Sports Med Arthrosc Rev. 2014;22(2):88-93. doi:10.1097/JSA.000000000000001 $\underline{5}$

15. Myers JB, Laudner KG, Pasquale MR, Bradley JP, Lephart SM. Glenohumeral range of motion deficits and posterior shoulder tightness in throwers with pathologic internal impingement. Am J Sports Med. 2006;34(3):385-391.

16. Tyler TF, Gleim GW. Quantification of posterior capsule tightness and motion loss in patients with shoulder impingement. Am J Sports Med. 2000;28(5):6.

17. Mine K, Takashi Nakayama, Milanese S, Grimmer K. Effectiveness of stretching on posterior shoulder tightness and glenohumeral internal-rotation deficit: A systematic review of randomized controlled trials. J Sport Rehabil. 2017;26(4):294-305.

18. Myers JB, Pasquale MR, Laudner KG, Sell TC, Bradley JP, Lephart SM. On-the-field resistancetubing exercises for throwers: An electromyographic analysis. J Athl Train. 2005;40(1):15-22. 
19. Borsa PA, Wilk KE, Jacobson JA, et al. Correlation of range of motion and glenohumeral translation in professional baseball pitchers. Am J Sports Med. 2005;33(9):1392-1399. doi:10.1177/036354650427349 $\underline{0}$

20. Borsa PA, Dover GC, Wilk KE, Reingold MM. Glenohumeral range of motion and stiffness in professional baseball pitchers. Med Sci Sports Exerc. 2006;38(1):21-26.

21. Greenberg EM, McClure P, Lawrence JTR, Fernandez-Fernandez A. Humeral retrotorsion and glenohumeral motion in youth baseball players compared with age-matched nonthrowing athletes. Am J Sports Med. 2017;45(2):454-461. doi:10.1177/036 $\underline{3546516676075}$

22. Chaabene H, Behm DG, Negra Y, Granacher U. Acute effects of static stretching on muscle strength and power: An attempt to clarify previous caveats. Front Physiol. 2019;10:1468. doi:10.3389/fphys.2019.0 $\underline{1468}$

23. Marek SM, Cramer JT, Fincher AL, et al. Acute effects of static and proprioceptive neuromuscular facilitation stretching on muscle strength and power output. J Athl Train. 2005;40(2):94-103.

24. Behm D, Chaouachi A, Behm DG. A review of the acute effects of static and dynamic stretching on performance. Eur J Appl Physiol. 2011;111(11):2633-2651. doi:10.1007/s00421-011-18 79-2

25. McMillian DJ, Moore JH, Hatler BS, Taylor DC. Dynamic vs. static-stretching warm up: the effect on power and agility performance. J Strength Cond Res. 2006;20(3):492-499.

26. Nelson AG, Kokkonen J, Arnall DA. Acute muscle stretching inhibits muscle strength endurance performance. J Strength Cond Res. 2005;19(2):338-343.

27. Young WB, Behm DG. Effects of running, static stretching and practice jumps on explosive force production and jumping performance. J Sports Med Phys Fitness. 2003;43(1):21-27.

28. Reid JC, Greene R, Young JD, Hodgson DD, Blazevich AJ, Behm DG. The effects of different durations of static stretching within a comprehensive warm-up on voluntary and evoked contractile properties. Eur J Appl Physiol. 2018;118(7):1427-1445. doi:10.1007/s00421-018-3874-3
29. Torres EM, Kraemer WJ, Vingren JL, et al. Effects of stretching on upper-body muscular performance. $J$ Strength Cond Res. 2008;22(4):1279-1285. doi:10.151 9/JSC.0b013e31816eb501

30. Knudson DV, Noffal GJ, Bahamonde RE, Bauer JA, Blackwell JR. Stretching has no effect on tennis serve performance. J Strength Cond Res. 2004;18(3):654-656. doi:10.1519/13553.1

31. Haag SJ, Wright GA, Gillette CM, Greany JF. Effects of acute static stretching of the throwing shoulder on pitching performance of national collegiate athletic association division III baseball players. J Strength Cond Res. 2010;24(2):452-457. doi:1 $\underline{0.1519 / J S C .0 b 013 e 3181 \mathrm{c} 06 \mathrm{~d} 9 \mathrm{c}}$

32. Cabido CET, Bergamini JC, Andrade AGP, Lima FV, Menzel HJ, Chagas MH. Acute effect of constant torque and angle stretching on range of motion, muscle passive properties, and stretch discomfort perception. J Strength Cond Res. 2014;28(4):1050. do i:10.1519/ISC.0000000000000241

33. Magnusson SP, Simonsen EB, Aagaard P, Gleim GW, McHugh MP, Kjaer M. Viscoelastic response to repeated static stretching in the human hamstring muscle. Scand J Med Sci Sports. 1995;5(6):342-347.

34. Taylor DC, Dalton JD Jr, Seaber AV, Garrett WE Jr. Viscoelastic properties of muscle-tendon units. The biomechanical effects of stretching. Am J Sports Med. 1990;18(3):300-309.

35. Amiri-Khorasani M, Abu Osman NA, Yusof A. Acute effect of static and dynamic stretching on hip dynamic range of motion during instep kicking in professional soccer players. J Strength Cond Res. 2011;25(6):1647. doi:10.1519/JSC.0b013e3181db9f41

36. Moreside JM, McGill SM. Hip joint range of motion improvements using three different interventions. J Strength Cond Res. 2012;26(5):1265-1273. doi:10.1519/ISC.0b013e31824f $\underline{2351}$

37. Gross ML, Flynn M, Sonzogni JJ. Overworked shoulders: managing injury of the proximal humeral physis. Phys Sportsmed. 1994;22(3):81-86. 